\title{
Study on Resource Management Based on Cloud Computing
}

\author{
Fu Gui FANG ${ }^{1, a}$ \\ ${ }^{1}$ Hexi University, 734000 Gansu China
}

\begin{abstract}
In view of that it is difficult to unify the resources management and the low system security in current cloud computing, the paper designs resource management system. First, it is the definition of cloud computing, architecture description of cloud services and cloud computing; followed by a detailed analysis of ant colony algorithm which is the core task scheduling in cloud computing; finally, it is the design framework and core code of resource management system based on cloud computing. This paper has a positive effect for big data staff and cloud computing researchers.

Key words. Cloud computing, Resource management, Task scheduling
\end{abstract}

\section{Introduction}

With the rapid development of information technology processes, network service requires large scale of resources and computing, which makes a separate computer cannot meet the needs of the current resource management. Cloud computing is the realization of commercialization network computing which is combined by parallel computing, network storage, load balancing, distributed computing and virtualization technology and the networks. As the characteristic of heterogeneity and distributivity, also the varied service types, when cloud computing manages resources, in order to meet the various needs of different users, it will have a huge scale of storage and calculation.

\section{Cloud computing}

\subsection{Definition of Cloud Computing}

In the era of information explosion today, it has become part of people's lives that we work in high-efficiency and low cost by use of internetwork communication for resources computing and storage. Along with the deepening of network applications, a lot of new concept and computational models such as on-demand computing, efficacy computing, grid computing which are proposed continuously and applied in the large-scale collaboration, cloud computing is presented in this case.

Since the introduction of cloud computing, experts and scholars at home and abroad proposed different definitions of cloud computing based on their own application scope and reality, but so far it does not have a clear definition. In this article, cloud computing is defined as a computing model which relies on the Internet, by increasing and using related network services

\footnotetext{
a Corresponding author: 931773740@qq.com
}

and less management costs, gets dynamic, a lot of computing resources for users to use seamlessly.

\subsection{Cloud Services}

At present, cloud computing can provide various types of services, but it can be summed up as three types: Laas (infrastructure as a service), Paas (Platform as a Service) and SaaS (Software as a service). With underlying hardware, the structure of these three kinds of services is shown in Figure 1:

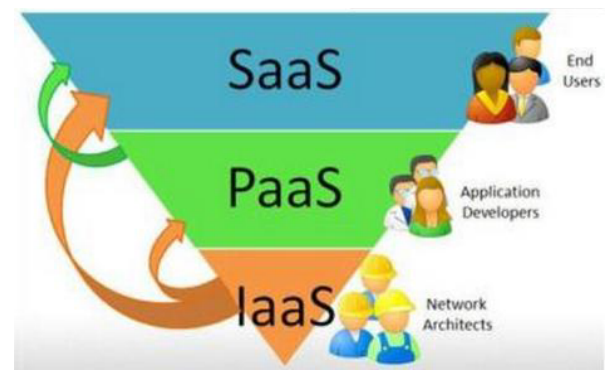

Figure 1 Structure of cloud services

\subsubsection{LaaS}

Related to the underlying hardware closely, the service of this mode provides storage, calculation and related infrastructure resources, its theoretical basis is virtual technology, and it will make the users feel no difference resource management when they use cloud computing.

\subsubsection{Paas}

This mode is a business model which provides development platform and server platform for developers, 
by loading some configuration for operating system platform, it provides service interface for secondary development, by eliminating infrastructure work management, it brings great convenience for the users.

\subsubsection{SaaS}

This mode mainly aims at end users, which is specially designed for specific services of network, it reduces unnecessary infrastructure configuration for end-users.

\subsection{System architecture of Cloud Computing}

After years of development, Cloud computing has gradually formed relatively complete system architecture, its system architecture is shown in Figure 2:

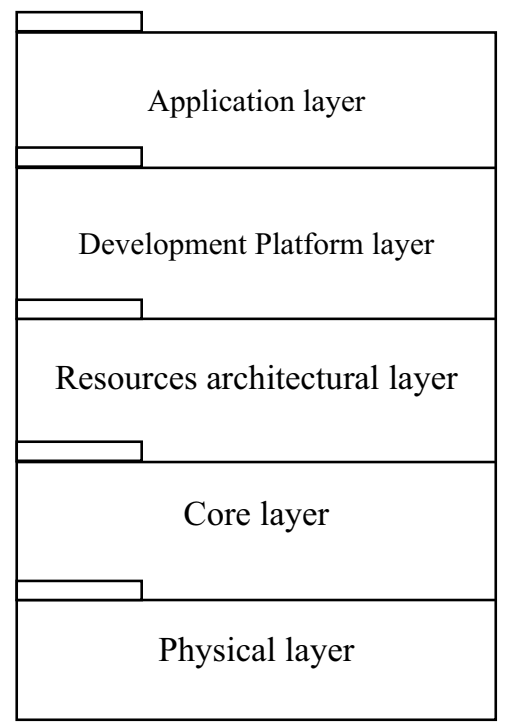

Figure 2. System architecture of cloud computing

As shown above, the entire system architecture is divided into five levels, of which the underlying physical layer is composed by the infrastructures distributing at different geographic locations; core layer manages physical resources, and provides abstract service of specific application for the upper layer; resource architecture layer ensures the reliable distributed communications and the construction of storage resources architecture system; development platform layer provides interface for specific application through API, which is the application platform of resource development; based on the development platform layer, application layer provides reliable quality service for the users.

\section{Task scheduling of Cloud computing}

There are many task scheduling algorithms in cloud computing, by comparison, ant colony algorithm has the advantages that it is easy to combine with other algorithms, so this article takes ant colony algorithm as the task scheduling algorithm of cloud computing.

\subsection{Principle of ant colony algorithm}

Ant colony algorithm was first proposed by Italian scholar Dorigo in 1992, it was a kind of bionic algorithm derived from nature. In nature, it was discovered that when ants look for food, they released a unique secretion along the way, which we called it pheromone, within a certain range, ant will decide its next route according to how much pheromones there are, when the longer the way was, the less pheromone was left, this will provide a reference to the ants back, when there were more pheromone left on a way, it showed that there were more ants had passed by, it had greater likelihood to find food on that way, and then it attracted different numbers of ants moving food along the best route.

Principle of ant colony algorithm is by use of ant groups transferring information in the path to achieve optimization. This algorithm has a lot of advantages including positive feedback mechanism, better robustness, self-organizing ability, good parallelism; but due to the short generating time, ant colony algorithm has some shortcomings in the application process, the main performance is slow convergence, long search time and ease of stagnant status.

\subsection{Task scheduling process based on ant colony algorithm}

\subsubsection{Mathematical model of ant colony algorithm}

Suppose that there are $\mathrm{m}$ ants to find $\mathrm{n}$ food nodes, between two food nodes the distance is dij, bi(t) is the number of ants located on Food node $i$ when $t$ time, then the total number of ants $m=\sum_{i=1}^{n} b i(t) \quad$ wherein ants leaving 0 pheromone at the first time on the road, after some time of searching food, the pheromone in each path is expressed as formula 1 :

$$
\tau_{i j}(t+1)=(1-\rho) \tau_{i j}(t)+\Delta \tau_{i j}(t)
$$

Formula 1

Where $\rho$ is the evaporation coefficient of pheromone, $\Delta \Gamma$ is the increased volume of pheromone.

The probability of Ant $\mathrm{k}$ moving from node $\mathrm{i}$ to node $\mathrm{j}$ is represented by Equation 2:

$$
p_{i j}^{k}= \begin{cases}\frac{\left[\tau_{i j}(t)\right]^{\alpha} \cdot\left[\eta_{i j}(t)\right]^{\beta}}{\left.\sum_{s \in \text { allowed }_{k}} \tau_{i s}(t)\right]^{\alpha} \cdot\left[\eta_{i s}(t)\right]^{\beta}} & \text { if } j \in \text { allowed }_{k} \\ 0 & \text { otherwise }\end{cases}
$$

Equation 2

The larger $\mathrm{P}$ is, the greater likelihood ant moves.

\subsubsection{Flow of algorithm}

Ants always tend to move on the way having more pheromone, by recording the nodes have been passed into the table $\mathrm{T}$, after entire discovery process, the value of pheromone is updated, by repeated execution till the final termination conditions, the particular algorithm folw is as follows: 
Step 1: Initialize the data, place $\mathrm{m}$ ants on $\mathrm{n}$ nodes randomly;

Step 2: Store initial node of all the ants into a specific table T;

Step 3: Determine whether the number of iterations has reached the set value, if it has reached, go to step 7, otherwise go to step 4;

Step 4: Calculate the probability of transfer by formula 2 above, and move to the corresponding node, modify the corresponding node value in table $\mathrm{T}$;

Step 5: Check if table $\mathrm{T}$ meets the condition, if not skip to step 4, if it meets go to step 6;

Step 6: Update the value of the pheromone, and empty table T, plus 1 to iteration value;

Step 7: Get the best value, ends.

\section{Resource Management System of Cloud Computing}

\subsection{Architecture Design of the System}

According to the architecture of cloud computing and the analysis of ant colony algorithm, resource management system of cloud computing designed has different rights management for users and administrators. It includes checking and applying status of VPS (virtual machine) host, basic operation(switch, restart, etc.) to VPS (virtual machine) host, checking the task application and task execution schedule, and submit computing tasks. The framework of its system is shown in Figure 3:

\section{2 system implementation}

In the system, ant colony algorithm is the core, in the process of searching nodes, it make ants find all of the nodes, wherein the core code is as follows:

$$
\text { if (e.Node.Tag! = null) }
$$

try

SearchKey = "';

string path $=$ e.Node.Tag.ToString ();

FileInfo file $=$ new FileInfo (path);

if ((file.Attributes \& FileAttributes.Directory)! =

0)

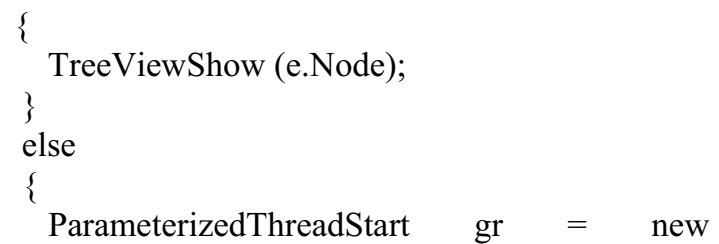
MessageBoxButtons.OK, MessageBoxIcon.Information);

\section{Summary}

This paper researches the resource management of cloud computing, so the resource management of cloud computing is the main content. This paper introduces ant colony algorithm which is the major algorithm in cloud computing, and designs out the framework. Due to limited space, this paper does not offer the specific function description and database design of the system completely, readers who are interested can do further research and discussion on this basis.

\section{References}

1. Li Xiaolong, Li Ting ,Research on resource management of cloud computing, Computer \& Telecommunication, 1,p.62-64(2010)

2. Yang Siwei, Gao Donghuai, Ning Yuwen, Research on network learning resource sharing based on Cloud Computing, The Chinese Journal of ICT in Education, 3,p.20-23(2012)

3. Aao li-bo, platform design of teaching resources sharing based on cloud Computing, Information Technology,10,p.46-48(2015)

4. Zhang Lei ,Cloud computing and information resource sharing management, Electronic Technology \& Software Engineering,4,p.21(2014)

5. Fan Guisheng, Yu Huiqun,Cloud computing security model and management, Microcomputer Applications, 1,p.1-3(2013)

6. Lv Yan,Research on human resource management information platform based on Cloud Computing, Management \& Technolosy of SME,20,p.182183(2015)

7. Jiang Zongpin, Luo Guofu, Liu Haidong,Design and implementation of logistics information platform based on Cloud Computing, Logistics Technology,13,p.256-258 (,2015)

8. Duan Zhongxiang, Construction of shared resource model of network platform based on Cloud Computing, Computer Engineering \& Software,5,p.119-121(2013)

9. Fang Jinming, The decision making system of virtual resource scheduling in cloud computing, Computer Measurement \& Control,12,p.2145-2148(2011)

10. Liu Guoying,Library resource management system in the era of cloud computing, Journal of Baoshan University,3,p.28-32(2015) 


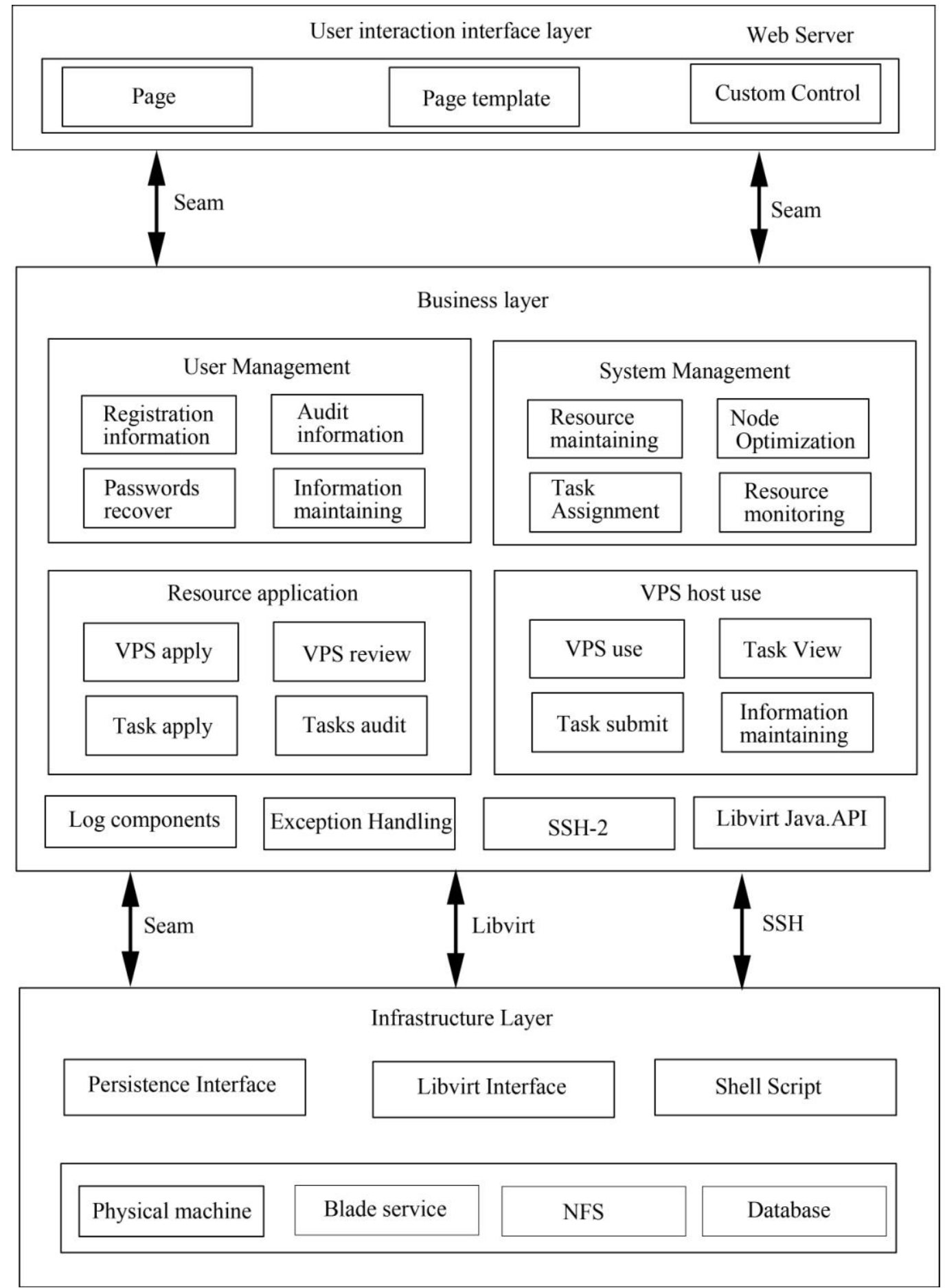

Figure 3. System Framework 